\title{
RAMSEY GRAPHS AND BLOCK DESIGNS. I
}

\author{
BY
}

\section{T. D. PARSONS}

ABSTRACT. This paper establishes a connection between a certain class of Ramsey numbers for graphs and the class of symmetric block designs admitting a polarity. The main case considered here relates the projective planes over Galois fields to the Ramsey numbers $R\left(C_{4}, K_{1, n}\right)=f(n)$. It is shown that, for every prime power $q, f\left(q^{2}+1\right)=q^{2}+q+2$, and $f\left(q^{2}\right)=q^{2}+q+1$.

1. Introduction. Let $A$ and $B$ be finite graphs. An $(A, B, m)$-graph is a graph $G$ with $m$ vertices such that $G$ contains no subgraph isomorphic to $A$ and the complement $\bar{G}$ of $G$ contains no subgraph isomorphic to $B$.

A well-known theorem of Ramsey [28] implies the existence of a positive integer $R(A, B)$ such that an $(A, B, m)$-graph exists if and only if $m<R(A, B)$. An $(A, B)$-graph is an $(A, B, m)$-graph with $m=R(A, B)-1$. The problem of determining the numbers $R(A, B)$ and the $(A, B)$-graphs for given $A, B$ is an area of extremal graph theory which has recently begun to develop rapidly. We refer to this area as "Ramsey graph theory".

The first results of Ramsey graph theory were for the cases where $A, B$ are complete graphs $K_{r}, K_{s}$. In 1935, Erdüs and Szekeres [15] gave estimates for the numbers $R\left(K_{r}, K_{s}\right)$. In 1955, Greenwood and Gleason [21] computed these numbers for the cases $(r, s)=(3,4),(3,5),(4,4)$; and in 1968, Graver and Yackel [20] computed the cases $(3,6),(3,7)$. No further nontrivial cases have been computed, although various bounds have been obtained.

It was preoccupation with the difficult $R\left(K_{r}, K_{s}\right)$ which delayed consideration of more general $R(A, B)$. The latter problem was defined first in 1966 by Gerencsér and Gyárfás [19], who computed all numbers $R\left(P_{m}, P_{n}\right)$, where $P_{m}$ denotes the path with $m$ vertices. However, the general problem was anticipated in a paper of Erdös [12] in 1947, in which the right upper bound for the numbers $R\left(P_{m}, K_{n}\right)$ is determined. Other general formulations were given independently by Cockayne [11] in 1970, and by Chvátal and Harary [7] in 1972.

In contrast to the case of the numbers $R\left(K_{r}, K_{s}\right)$, many nontrivial results have now been obtained for the numbers $R(A, B)$. Let $C_{m}$ denote the cycle with 
$m$ vertices, and $K_{m, n}$ the complete bipartite graph on sets of $m$ and $n$ vertices. The numbers $R(A, B)$ have been computed for all pairs $(A, B)=\left(C_{m}, C_{n}\right)$, $\left(P_{m}, P_{n}\right),\left(P_{m}, C_{n}\right),\left(P_{m}, K_{1, n}\right),\left(K_{1, m}, K_{1, n}\right),\left(K_{m}, K_{1, n}\right)$. Rather than discuss these and other results, we refer the reader to our bibliography, which will be necessarily incomplete due to the rapid rate of new results in this area.

This paper begins study of the numbers $R\left(K_{2, m}, K_{1, n}\right)$, which are closely related to $(v, k, \lambda)$-block designs admitting a polarity, and to variants of the "Friendship Theorem" [14], [25]. The $\left(K_{2, m}, K_{1, n}\right)$-graphs contrast with most other classes of $(A, B)$-graphs previously constructed, the latter being typically disjoint unions of complete graphs (or complements of such unions).

The main part of the paper is devoted to the case $m=2, n=q^{2}+1$ where $q$ is a prime power. In this case, the corresponding block designs are the Singer $\left(q^{2}+q+1, q+1,1\right)$ difference sets corresponding to projective planes over the finite fields $G F(q)$.

The concluding section of the paper states the way in which this result generalizes to other block designs, and in particular to $(v, k, \lambda)$-difference sets in finite abelian groups. Our general results will appear elsewhere as Ramsey graphs and block designs, the forerunner of the present paper which is itself part I of several specialized applications.

2. The numbers $R\left(C_{4}, K_{1, n}\right)$. Let $G$ be a finite graph with no loops or multiple edges, with vertex set $V G$ and edge set $E G$. Let $m=|V G|, \Delta(v)=$ $\{w \in V G \mid v w \in E G\}$ be the set of vertices adjacent to the vertex $v$ in $G, \delta(v)=$ $|\Delta(v)|$ be the valence of vertex $v$ in $G$, and $\delta(G)$ the least valence of $G$. If $v$ and $w$ are distinct vertices, we let $\delta(v, w)=|\Delta(v) \cap \Delta(w)|$, and dist $(v, w, G)$ be the length of a shortest path in $G$ from $v$ to $w$ (if one exists).

Bars denote analogous terms for the complement $\bar{G}$ of $G$. Thus $\bar{\delta}(v)$ is the valence of $v$ in $\bar{G}$. We often abbreviate $V G, E G, \delta(G), E(\bar{G}), \delta(\bar{G})$ by $V, E, \delta, \bar{E}$, $\bar{\delta}$. When $G$ is clear from context, we write $u \sim v$ for "vertex $u$ is adjacent to vertex $v$ ", that is, for $u v \in E G$.

If $H$ is a graph, then $G \supset H$ (respectively, $G \supset H$ ) means $G$ contains (does not contain) a subgraph isomorphic to $H$. For each positive integer $n$, we let

$$
F_{n}=\left\{G \mid G \supset C_{4} \text { and } \bar{G} \supset K_{1, n}\right\}
$$

be the set of graphs not containing a cycle of length 4 and whose complement has no vertex of valence $n$ or more.

Lemma 1. Let $n>1$. If $G \in F_{n}$, then $m \leqslant n+\sqrt{n-1}+1$. If also $\delta>$ $m-n$, then $m \leqslant n+\sqrt{n-2}$.

PROof. Since $n>1, n+\sqrt{n-1}+1 \geqslant n+2$, so we may assume that 
$m \geqslant n+3>4$. Let $\left\{v_{1}, v_{2}, \cdots, v_{m}\right\}=V$ and $\delta_{i}=\delta\left(v_{i}\right)$. Since $\delta_{i}+\bar{\delta}_{i}=m-1$ and $\bar{\delta}_{i} \leqslant n-1$, we have $\delta_{i} \geqslant m-n \geqslant 3$, so $\delta \geqslant m-n$. Since $G \supset C_{4}$, if $i \neq j$ then there is at most one vertex $v_{k}$ adjacent to both $v_{i}$ and $v_{j}$ in $G$. For each $k$, there are $\left({ }_{2}^{\delta^{k}}\right)$ pairs $v_{i}, v_{j}(i \neq j)$ with both $v_{i}$ and $v_{j}$ adjacent to $v_{k}$ in $G$. Thus

$$
\sum_{k=1}^{m}\left(\begin{array}{l}
\delta_{k} \\
2
\end{array}\right) \leqslant\left(\begin{array}{l}
m \\
2
\end{array}\right) .
$$

If equality held here, then for every pair $v_{i}, v_{j}(i \neq j)$ of vertices there would exist a unique vertex $v_{k}$ such that both $v_{i} \sim v_{k}$ and $v_{j} \sim v_{k}$. Then the "Friendship Theorem" [14, Theorem 6], [25] would imply that some $\delta_{i}=2$, contradicting $\delta_{i} \geqslant m-n \geqslant 3$. Thus

$$
\sum_{k=1}^{m}\left(\begin{array}{l}
\delta_{k} \\
2
\end{array}\right)<\left(\begin{array}{l}
m \\
2
\end{array}\right)
$$

so that $\delta(\delta-1)<m-1$. Since $\delta$ is an integer, $\delta(\delta-1) \leqslant m-2$.

Now $\delta \geqslant m-n \geqslant 3$, and $x(x-1)$ is an increasing function of $x$ for $x>$ $1 / 2$, so $\delta(\delta-1) \geqslant(m-n)(m-n-1)$. It follows that $m-2 \geqslant(m-n)(m-n-1)$, and this implies by a simple calculation that $m \leqslant n+\sqrt{n-1}+1$.

If $\delta>m-n$, then $\delta \geqslant m-n+1$ and similar reasoning shows that $m \leqslant n+$ $\sqrt{n-2}$.

REMARK. If $n=k^{2}$ is a square, the lemma says that $m<k^{2}+k+1$ so $m \leqslant k^{2}+k=n+\sqrt{n}$. Theorem 2 below shows that this bound is attained whenever $k$ is a prime power, thus Lemma 1 is a best possible bound.

If $n$ is not a square, then the lemma gives that $m \leqslant n+[\sqrt{n}]+1$. This bound is attained when $n=7$ by the Petersen graph, proving $R\left(C_{4}, K_{1,7}\right)=11$. The author does not yet know whether $m=n+[\sqrt{n}]+1$ can occur for infinitely many $n$ not squares. Our results below show that $m \leqslant n+[\sqrt{n}]$ whenever $n=$ $q^{2}+1$ and $q$ is a positive integer.

LEMMA 2. Let $q \geqslant 2$ be an integer. Let $n=q^{2}+1, G \in F_{n}$, and $m=q^{2}$ $+q+2$. Then the following are true:

(1) $G$ is regular of valence $q+1$.

(2) For every $v \in V$ there is a unique $v^{*} \in V \backslash\{v\}$ such that $\delta\left(v, v^{*}\right)=0$. If $w \in V \backslash\left\{v, v^{*}\right\}$, then $\delta(v, w)=1$. Thus $\left(v^{*}\right)^{*}=v$.

(3) If $q$ is odd, then there is a map $f: \Delta(v) \rightarrow \Delta(v)$ such that $w \sim f(w)$ and $f^{2}(w)=w$ for all $w \in \Delta(v)$. Also, $\operatorname{dist}\left(v, v^{*}, G\right)=3$, and $\operatorname{dist}\left(w, v^{*}, G\right)=2$ for all $w \in \Delta(v)$.

(4) If $q$ is even, then $v^{*} \in \Delta(v)$ and there is a map $f: \Delta(v) \backslash\left\{v^{*}\right\} \rightarrow$ $\Delta(v) \backslash\left\{v^{*}\right\}$ such that $w \sim f(w)$ and $f^{2}(w)=w$ for all $w \in \Delta(v) \backslash\left\{v^{*}\right\}$. 
Proof. By Lemma 1 , if $\delta>m-n$, then

$$
q^{2}+q+2=m \leqslant n+\sqrt{n-2}=q^{2}+1+\sqrt{q^{2}-1}<q^{2}+q+1,
$$

a contradiction. Since $\bar{G} \not K_{1, n}$, we have $\delta \geqslant m-n$. Thus $\delta=m-n=\left(q^{2}+\right.$ $q+2)-\left(q^{2}+1\right)=q+1$.

Suppose some vertex $v$ of $G$ has valence $\delta(v)=r$ where $r>q+1$. Let $\Delta(v)=\left\{v_{1}, v_{2}, \cdots, v_{r}\right\}$. If there were distinct $i, j, k \leqslant r$ such that $v_{i} \sim v_{j}$ and $v_{i} \sim v_{k}$, then $G$ would contain the $C_{4}\left\langle v, v_{j}, v_{i}, v_{k}, v\right\rangle$. Thus, for each $i, 1 \leqslant i \leqslant r$, there is at most one $j, 1 \leqslant j \leqslant r$, with $v_{i} \sim v_{j}$.

For $1 \leqslant i \leqslant r$, let $A_{i}=\left\{w \mid w \in \Delta\left(v_{i}\right), w \neq v\right.$, and $\left.w \notin \Delta(v)\right\}$. Suppose $i \neq j$ and $w \in A_{i} \cap A_{j}$. Then $\left\langle v, v_{i}, w, v_{j}, v\right\rangle$ would be a $C_{4}$ in $G$. Thus $A_{1}, A_{2}, \cdots$, $A_{r}$ are mutually disjoint. Since there is at most one $z \in \Delta(v)$ with $z \sim v_{i}$, we have either $A_{i}=\Delta\left(v_{i}\right) \backslash\{v, z\}$ if $z$ exists, or $A_{i}=\Delta\left(v_{i}\right) \backslash\{v\}$ if no such $z$ exists. Thus, for $1 \leqslant i \leqslant r,\left|A_{i}\right| \geqslant \delta-2=q-1$. Now $V$ contains the disjoint sets $\{v\}$, $\Delta(v), A_{1}, \cdots, A_{r}$ so

$$
q^{2}+q+2=|V| \geqslant 1+r+r(q-1)=1+r q \geqslant 1+(q+2) q=q^{2}+2 q+1 \text {, }
$$

so $1 \geqslant q$, a contradiction. It follows that $G$ is regular of valence $q+1$.

Let $v \in V$ and $\Delta(v)=\left\{v_{1}, v_{2}, \cdots, v_{q+1}\right\}$. Define $A_{i}$, for $1 \leqslant i \leqslant q+1$, as was done above. As before, each $v_{i}$ is adjacent to at most one other vertex $v_{j}$ for $1 \leqslant i, j \leqslant q+1$. Thus

$$
\left|A_{i}\right|= \begin{cases}q-1, & \text { if there is a } v_{j} \sim v_{i} \text { with } j \leqslant q+1, \\ q, & \text { otherwise. }\end{cases}
$$

As before, the sets $\{v\}, \Delta(v), A_{1}, \cdots, A_{q+1}$ are disjoint, so $q^{2}+q+2=|V|$ $\geqslant \sum_{i=1}^{q+1}\left|A_{i}\right|+(q+1)+1 ;$ thus $q^{2} \geqslant \sum_{i=1}^{q+1}\left|A_{i}\right| \geqslant(q+1)(q-1)=q^{2}-1$.

It follows that there is at most one index $i(1 \leqslant i \leqslant q+1)$ such that $\left|A_{i}\right|=q$. We may renumber $v_{1}, v_{2}, \cdots, v_{q+1}$ if necessary so that $\left|A_{1}\right| \geqslant\left|A_{2}\right|$ $=\left|A_{3}\right|=\cdots=\left|A_{q+1}\right|=q-1$, which we now assume.

Case 1. Suppose $\left|A_{1}\right|=q$. Then, for all $w \in \Delta(v), v_{1}$ is not adjacent to $w$ in $G$, so $v_{1} \notin \Delta\left(v_{i}\right)$ for $2 \leqslant i \leqslant q+1$. If $2 \leqslant i \leqslant q+1$, then $\left|A_{i}\right|=q-1$, so there is a unique $w \in \Delta(v)$ with $w \sim v_{i}$; but $w \neq v_{1}$, so $w=v_{j}$ for some $j \neq i$ with $2 \leqslant j \leqslant q+1$. Define $f: \Delta(v) \backslash\left\{v_{1}\right\} \rightarrow \Delta(v) \backslash\left\{v_{1}\right\}$ by $f\left(v_{i}\right)=v_{j}$, where $v_{i} \sim v_{j}$. Then $f\left(v_{i}\right) \sim v_{i}$ so $f\left(v_{i}\right) \neq v_{i}$; and obviously $f\left(v_{j}\right)=v_{i}$ so $f^{2}\left(v_{i}\right)=v_{i}$. It follows that $q$ must be even. Since $\{v\}, \Delta(v), A_{1}, \cdots, A_{q+1}$ are disjoint sets whose cardinalities sum to $1+(q+1)+q+q(q-1)=q^{2}+q+2=|V|$, we must have that

$$
V=\{v\} \cup \Delta(v) \cup A_{1} \cup \cdots \cup A_{q+1} .
$$

If $2 \leqslant i \leqslant q+1$, then $\left\langle v, f\left(v_{i}\right), v_{i}\right\rangle$ is a path of length 2 from $v$ to $v_{i}$ in $G$. If 
$w \in A_{i}$ for $1 \leqslant i \leqslant q+1$, then $\left\langle v, v_{i}, w\right\rangle$ is a path of length 2 from $v$ to $w$. Suppose $\left\langle v, w, v_{1}\right\rangle$ is a path of length 2 in $G$; then $v \sim w$ and $w \sim v_{1}$, so $v_{1}$ is in $\Delta\left(v_{i}\right)$ for some $i$, a contradiction. Thus $v^{*}=v_{1}$ is the unique vertex in $V \backslash\{v\}$ for which there is no path $\left\langle v, w, v_{1}\right\rangle$ of length 2 in $G$, and we have $v^{*} \in \Delta(v)$.

Case 2. Suppose $\left|A_{1}\right|=q-1$. Let $B=\{v\} \cup \Delta(v) \cup A_{1} \cup \cdots \cup A_{q+1}$. Then $|B|=1+(q+1)+(q+1)(q-1)=q^{2}+q+1=|V|-1$, so there is a unique $v^{*} \in V \backslash B$.

For each $i, 1 \leqslant i \leqslant q+1,\left|A_{i}\right|=q-1$, so there is for each $v_{i}$ a unique $v_{j}$ such that $j \neq i, 1 \leqslant j \leqslant q+1$, and $v_{i} \sim v_{j}$. Define $f: \Delta(v) \rightarrow \Delta(v)$ by $f\left(v_{i}\right)=$ $v_{j}$ where $v_{i} \sim v_{j}$. Then $f\left(v_{i}\right) \neq v_{i}$ and $f\left(v_{j}\right)=v_{i}$ if and only if $f\left(v_{i}\right)=v_{j}$, so $f^{2}\left(v_{i}\right)=v_{i}$. It follows that $q+1$ is even, hence $q$ is odd.

If $w \in \Delta(v)$, then $\langle v, f(w), w\rangle$ is a path of length 2 in $G$. If $1 \leqslant i \leqslant q+1$ and $w \in A_{i}$, then $\left\langle v, v_{i}, w\right\rangle$ is a path of length 2 in $G$. Suppose $\left\langle v, w, v^{*}\right\rangle$ is a path of length 2 in $G$; then $w \sim v$ so $w=v_{i}$ for some $i, 1 \leqslant i \leqslant q+1$, and we have $v^{*} \in \Delta\left(v_{i}\right), v^{*} \neq v$, and $v^{*} \notin \Delta(v)$; so $v^{*} \in A_{i}$, a contradiction.

Thus $v^{*}$ is the unique vertex in $V \backslash\{v\}$ for which there is no path of length 2 from $v$. Since $v^{*} \notin \Delta(v)$, and $\delta\left(v, v^{*}\right)=0$, the distance from $v$ to $v^{*}$ in $G$ is at least 3. But $v^{*}$ has valence $q+1$ in $G$, so $v^{*} \sim z$ for some $z \in B$, and since $\operatorname{dist}\left(v, v^{*}, G\right) \geqslant 3, z \in A_{i}$ for some $i$; hence $\operatorname{dist}\left(v, v^{*}, G\right)=3$.

If $v^{*}$ were adjacent to two vertices $w, z$ of $A_{i}$, then $\left\langle v_{i}, w, v^{*}, z, v_{i}\right\rangle$ would be a $C_{4}$ in $G$, so actually $v^{*}$ is adjacent to exactly one vertex $z_{i}$ in each set $A_{i}$ for $1 \leqslant i \leqslant q+1$. This implies $\operatorname{dist}\left(v_{i}, v^{*}, G\right)=2$.

Since $G \supset C_{4}$, there is at most one path of length 2 joining any two vertices of $G$. Thus $\delta(v, w)=1$ if $v \neq w \neq v^{*}$.

Since Case 1 implies $q$ is even and Case 2 implies $q$ is odd, we must have either that $q$ is even and Case 1 holds for every $v \in V$, or $q$ is odd and Case 2 holds for every $v \in V$. This completes the proof of the lemma.

LeMma 3. Let $q \geqslant 2$ be an even integer. Let $n=q^{2}+1$ and $G \in F_{n}$. Then $m<q^{2}+q+2$.

Proof. By Lemma $1, m \leqslant q^{2}+q+2$. Suppose that $m=q^{2}+q+2$. Then, by Lemma $2, G$ is regular of valence $q+1$ and for every $v \in V$ there exists a unique $v^{*} \in \Delta(v)$ such that $\delta\left(v, v^{*}\right)=0$, while $\delta(v, w)=1$ for every $w \in$ $V \backslash\left\{v, v^{*}\right\}$. It follows that the set $V$ with relation $\sim$ ("adjacency in $G^{\prime \prime)}$ is a nontrivial "homogeneous friendship set" in the sense of H. L. Skala [34], and Skala has proved [34, Theorem 2; actually the argument at the beginning of the proof suffices] in this case that $v \sim w$ implies there is no path of length 2 from $v$ to $w$. But if $v \in V,|\Delta(v)|=q+1 \geqslant 3$; so we may choose $w \in \Delta(v) \backslash\left\{v^{*}\right\}$. Then $v \sim w$ and by Lemma 2 there is a path of length 2 from $v$ to $w$, contradicting Skala's theorem. It follows that $m<q^{2}+q+2$. 
Lemma 4. Let $q \geqslant 2, m=q^{2}+q+2$, and $n=q^{2}+1$. If there exists $a$ graph $G$ with $m$ vertices such that $G \in F_{n}$, then $q=5$.

Proof. By Lemma 3, $q$ must be odd. Suppose $G \in F_{n}$. By Lemma 2, we may number the vertices $v_{1}, v_{2}, \cdots, v_{m}$ of $G$ such that $v_{1}^{*}=v_{2}, v_{3}^{*}=v_{4}$, $\cdots, v_{m-1}^{*}=v_{m}$. Let $M$ be the matrix of order $m$ defined by

$$
M_{i j}= \begin{cases}1, & \text { if } v_{i} \sim v_{j} \text { in } G, \\ 0, & \text { otherwise. }\end{cases}
$$

Then $M$ is symmetric, and by Lemma $2, M$ has exactly $q+1$ nonzero entries in each row. Let $J$ be the order $m$ matrix with all entries $1, J^{*}$ the $m \times 1$ column vector with all entries $1, I$ the order $m$ identity matrix, and let $A$ be the order $m$ matrix composed of $m / 2$ blocks of the nontrivial order 2 permutation matrix down the main diagonal and zero entries elsewhere, that is

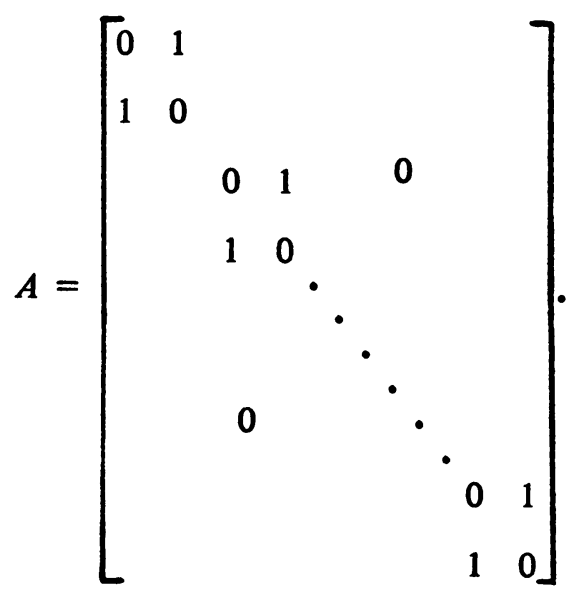

Lemma 2 then implies $M^{2}=q I+J-A$, since $\left(M^{2}\right)_{i i}$ is the valence of vertex $v_{i}$, and for $i \neq j,\left(M^{2}\right)_{i j}$ is the number of paths of length 2 from $v_{i}$ to $v_{j}$, and this number is 1 if $v_{j} \neq v_{i}^{*}$ and 0 if $v_{j}=v_{i}^{*}$.

Since $M J^{*}=(q+1) J^{*}, q+1$ is an eigenvalue and $J^{*}$ a corresponding eigenvector of $M$. Since $\operatorname{Trace}(M)=0, M$ has other eigenvalues. Let $\mu$ be an eigenvalue of $M$ such that $\mu \neq q+1$, and let $X$ be an eigenvector of $M$ for the eigenvalue $\mu$. Then $X$ is orthogonal to $J^{*}$ so $0=\left(J^{*}\right)^{T} X$, thus $J X=0$. It follows that $\mu^{2} X=M^{2} X=(q I+J-A) X=q X-A X$, so $A X=\left(q-\mu^{2}\right) X$; since $X \neq 0, q-\mu^{2}$ is an eigenvalue of $A$. But the eigenvalues of $A$ are obviously 1 and -1 , so $\mu^{2}=q+1$ or $\mu^{2}=q-1$. This implies that the only possible eigenvalues of $M$ are $q+1, \sqrt{q+1},-\sqrt{q+1}, \sqrt{q-1},-\sqrt{q-1}$. Of course, the possible eigenvalues of $M^{2}$ are then $(q+1)^{2}, q+1, q-1$. We first compute the dimensions of the eigenspaces of the matrix $M^{2}$. 
By elementary row operations, it is easy to show that the $m / 2$ vectors $(0,0,1,1, \cdots, 1),(1,1,0,0,1,1, \cdots, 1),(1,1,1,1,0,0,1,1, \cdots, 1)$, $\cdots,(1,1, \cdots, 1,0,0)$ are a basis for the row-space of the matrix $M^{2}-$ $(q+1) I$, so that the dimension of the eigenspace of $M^{2}$ corresponding to the eigenvalue $(q+1)$ of $M^{2}$ is $m / 2$.

Similarly, the vectors $(2,0,1,1, \cdots, 1),(0,2,1,1, \cdots, 1),(0,0,-1$, $-1,0,0, \cdots, 0),(0,0,0,0,-1,-1,0,0, \cdots: \cdots, 0), \cdots,(0,0, \cdots, 0$, $-1,-1)$ are easily seen to be a basis for the row-space of $M^{2}-(q-1) I$, which thus has rank $(m+2) / 2$ and so has nullity $(m-2) / 2$. Therefore the dimension of the eigenspace of $M^{2}$ corresponding to its eigenvalue $(q-1)$ is $(m-2) / 2$. Since $m / 2+(m-2) / 2=m-1$, the dimension of the eigenspace of $M^{2}$ corresponding to its remaining eigenvalue $(q+1)^{2}$ is 1 . Thus the eigenspace of $M$ corresponding to the eigenvalue $(q+1)$ of $M$ has dimension 1 .

Let $a, b, c, d$ be the multiplicities of the eigenvalues $\sqrt{q+1},-\sqrt{q+1}$, $\sqrt{q-1},-\sqrt{q-1}$ of $M$. It follows from the above remarks that $a+b=m / 2$ and $c+d=(m-2) / 2$. Since $\operatorname{Trace}(M)=0$, we have $q+1+(a-b) \sqrt{q+1}+$ $(c-d) \sqrt{q-1}$ is zero. Now $a-b=2 a-m / 2$ and $c-d=2 c-(m-2) / 2$, so we have

$$
(q+1)+(2 a-m / 2) \sqrt{q+1}+(2 c-(m-2) / 2) \sqrt{q-1}=0 .
$$

Since $q \geqslant 2$, we easily see that exactly one of the following two conditions holds:

(1) $q+1$ is a square and $2 c-(m-2) / 2=0$, or

(2) $q-1$ is a square and $2 a-m / 2=0$.

Suppose (1) holds. Since $q$ is odd, $q+1$ is an even square, so $q+1=4 k^{2}$ for some positive integer $k$. Dividing (*) by $\sqrt{q+1}$, we get $\sqrt{q+1}+(2 a-m / 2)$ $=0$, so $2 k+2 a=\sqrt{q+1}+2 a=m / 2$, thus $m / 2$ is even. But $q=4 k^{2}-1 \equiv 3$ $(\bmod 4)$, so $m=q^{2}+q+2 \equiv 2(\bmod 4)$, hence $m / 2 \equiv 1$ or $3(\bmod 4)$ so $m / 2$ is odd, a contradiction.

We conclude (1) does not hold, so (2) must. Since $q-1$ is then an even square, $\sqrt{q-1}=2 k$ for some positive integer $k$. Then $(*)$ and (2) imply that $q+1=((m-2) / 2-2 c) \sqrt{q-1} \equiv 0(\bmod 2 k)$ since $(m-2) / 2-2 c$ is an integer. But $q+1=4 k^{2}+2 \equiv 2(\bmod 2 k)$, so $2 \equiv 0(\bmod 2 k)$ so $k=1$. Thus $q=4 k^{2}$ $+1=5$, proving the lemma.

LEMma 5. There is no graph $G$ with 32 vertices in the class $F_{26}$.

Proof. This is the case $q=5$ not decided by the previous lemma.

Suppose such a graph $G$ exists. We may apply Lemma 2 with $q=5$. Following the notations of Lemma 4, we have that the adjacency matrix $M$ of $G$ obeys the equation $M^{2}=5 I+J-A$, where these matrices are of order 32 . 
Since $M J=6 J=J M$, we have

$$
\begin{aligned}
5 M+6 J-M A & =5 M+M J-M A=M(5 I+J-A)=M M^{2} \\
& =M^{2} M={ }^{\circ}(5 I+J-A) M=5 M+6 J-A M ;
\end{aligned}
$$

therefore $M A=A M$. But $A$ is a permutation matrix obeying $A=A^{T}=A^{-1}$, so $M=A M A^{T}$. Clearly $\left(A M A^{T}\right)_{i j}$ is 1 if $v_{i}^{*} \sim v_{j}^{*}$ and is 0 otherwise.

Thus $M=A M A^{T}$ implies $v_{i} \sim v_{j}$ if and only if $v_{i}^{*} \sim v_{j}^{*}$, so the map $v \mapsto v^{*}$ is an automorphism of $G$.

It is now a straightforward but tedious exercise to use the "local structure" of $G$ given by Lemma 2 , and to apply the automorphism $v \mapsto v^{*}$ to show that $G$ cannot exist. The details are repetitious and uninteresting, and will not be given here.

LEMma 6. Let $q=p^{s}$, where $p$ is a prime and $s$ is a positive integer. Then there exists a graph $G$ with $q^{2}+q+1$ vertices such that $G \perp C_{4}$ and $\bar{G} D$ $K_{1, q^{2}+1}$.

Proof. Let $F=G F(q)$ be the Galois field with $q$ elements. Define an equivalence relation on $F^{3} \backslash\{(0,0,0)\}$ by letting $(a, b, c) \equiv\left(a^{\prime}, b^{\prime}, c^{\prime}\right)$ if there is a nonzero $\lambda \in F$ such that $(\lambda a, \lambda b, \lambda c)=\left(a^{\prime}, b^{\prime}, c^{\prime}\right)$. Let $[a, b, c]$ denote the equivalence class of $(a, b, c)$, and let $V$ be the set of all equivalence classes. For $[a, b, c] \in V$, let $L[a, b, c]=\{[x, y, z] \in V \mid a x+b y+c z=0\}$.

Define a graph $G$ with vertex set $V$ by letting $[a, b, c] \sim[x, y, z]$ if $[a, b, c]$ $\neq[x, y, z]$ and $[x, y, z] \in L[a, b, c]$. Clearly, $|V|=q^{2}+q+1$, and $|L[a, b, c]|$ $=q+1$. Thus if $[a, b, c]$ obeys $a^{2}+b^{2}+c^{2} \neq 0$, then $[a, b, c] \sim[x, y, z]$ for all $[x, y, z]$ in $L[a, b, c]$, so $[a, b, c]$ has valence $q+1$ in $G$. On the other hand, if $a^{2}+b^{2}+c^{2}=0$, then $[a, b, c] \sim[x, y, z]$ for $[x, y, z] \in$ $L[a, b, c] \backslash\{[a, b, c]\}$ so $[a, b, c]$ has valence $q$ in $G$. [It is not difficult to show that there are exactly $q+1$ vertices $[a, b, c]$ of $G$ which have valence $q$.] It follows that the maximum valence of the complementary graph $\bar{G}$ is $q^{2}$, so $\bar{G} \not$ $K_{1, q^{2}+1}$.

Suppose $\langle[a, b, c],[x, y, z],[d, e, f],[u, v, w],[a, b, c]\rangle$ is a cycle of length 4 in $G$. Since $[a, b, c] \neq[d, e, f]$, the vectors $(a, b, c)$ and $(d, e, f)$ are linearly independent in $F^{3}$. But we have

$$
\begin{array}{ll}
a x+b y+c z=0, & d x+e y+f z=0, \\
a u+b v+c w=0, & d u+e v+f w=0,
\end{array}
$$

so there exists $\lambda \in F$ such that $(u, v, w)=\lambda(x, y, z)$ in $F^{3}$. Since $(u, v, w) \neq$ $(0,0,0)$, we have $\lambda \neq 0$ so $[u, v, w]=[x, y, z]$, a contradiction. Thus $G$ contains no $C_{4}$, completing the proof of the lemma.

REMARK. The above construction is due to Erdös, Rényi, and Sós [14], and independently to Brown [3]. It is worth noting that this construction provides 
examples of "nonhomogeneous friendship sets" (see Skala [34]) of degree $q+1$ for all prime powers $q$.

Indeed, suppose $[a, b, c]$ and $[x, y, z]$ are distinct points of $G$ not joined by a path of length 2 in $G$. The equations $a u+b v+c w=0, x u+y v+z w=0$ have a nontrivial solution $(u, v, w)$ in $F^{3}$. If $[u, v, w]$ were distinct from both $[a, b, c]$ and $[x, y, z]$, then $\langle[a, b, c],[u, v, w],[x, y, z]\rangle$ would be a path of length 2 in $G$, contrary to hypothesis. Thus either $[u, v, w]=[a, b, c]$ or $[u, v, w]$ $=[x, y, z]$, so $a x+b y+c z=0$, thus $[a, b, c] \sim[x, y, z]$ in $G$. This shows that $G$ (that is, $V$ with the relation $\sim$ ) is a nonhomogeneous friendship set of degree $q+1$, according to the definition given by Skala [34].

These graphs are a special case of the " $(v, k, \lambda)$-polarity graphs" we mention in the next section. We now show how they correspond to certain Ramsey numbers.

THEOREM 1. Let $f(n)=R\left(C_{4}, K_{1, n}\right)$. Then $f(n) \leqslant n+\sqrt{n-1}+2$ for all $n \geqslant 2$, and $f\left(q^{2}+1\right) \leqslant q^{2}+q+2$ for all $q \geqslant 1$.

If $q=p^{s}$, where $p$ is a prime and $s$ is a nonnegative integer, then $f\left(q^{2}+1\right)$ $=q^{2}+q+2$.

Proof. Let $G \in F_{n}$ have $m$ vertices, where $m$ is largest possible. Then $m=$ $f(n)-1$. Therefore $f(n) \leqslant n+\sqrt{n-1}+2$ for $n>1$ was proved in Lemma 1 , and $f\left(q^{2}+1\right) \leqslant q^{2}+q+2$ for all $q \geqslant 2$ was proved in Lemmas 4 and 5 . Trivially, $f(2)=4$ when $q=1$. If $q=p^{s}$ where $p$ is a prime and $s$ is a positive integer, $f\left(q^{2}+1\right)>q^{2}+q+1$ follows from Lemma 6 . This completes the proof of the theorem.

The author and S. L. Lawrence have jointly established the following theorem, which shows that the bound provided by Lemma 1 is attained infinitely often.

THEOREM 2. If $q=p^{s}$ where $p$ is a prime and $s$ is a positive integer, then $f\left(q^{2}\right)=q^{2}+q+1$.

PROof. Let $G$ be the graph constructed in Lemma 6 corresponding to the prime power $q$. It follows from the Friendship Theorem (stated in the next section) that $G$ has at least one vertex of valence $q$. In fact, the remarks immediately following the proof of Lemma 6 show that if $[a, b, c]$ and $[x, y, z]$ are distinct points of $G$ not joined by a path of length 2 in $G$, then either $a^{2}+b^{2}+c^{2}=0$ or $x^{2}+y^{2}+z^{2}=0$, so that either $[a, b, c]$ or $[x, y, z]$ has valence $q$ in $G$. It must be the case that some pair of vertices of $G$ is not joined by a path of length 2, otherwise the Friendship Theorem would imply that $G$ has a vertex of valence $q^{2}+q$, contradicting the fact that all valences in $G$ are $q$ or $q+1$.

We now show that no two $q$-valent vertices are adjacent in $G$. Suppose that $[a, b, c]$ and $[x, y, z]$ are distinct vertices of valence $q$ in $G$. Then the vectors $(a, b, c)$ and $(x, y, z)$ are linearly independent in $F^{3}$. Therefore, the subspace $S$ 
of $F^{3}$ consisting of all vectors $(u, v, w)$ such that $a u+b v+c w=0$ and $x u+y v$ $+z w=0$ is 1 -dimensional. If $[a, b, c]$ and $[x, y, z]$ were adjacent in $G$, then both $(a, b, c)$ and $(x, y, z)$ would be in $S$, a contradiction.

Now let $v$ be a vertex of valence $q$ in $G$. Let $H$ be the graph obtained from $G$ by deleting the vertex $v$ and all edges incident to it. Since $v$ was adjacent in $G$ only to vertices of valence $q+1$, the least valence of $H$ is $q$. Since $H$ has $q^{2}+q$ vertices, the maximum valence in the complementary graph $\bar{H}$ is $q^{2}-1$. Since $H$ has no cycle of length $4, f\left(q^{2}\right)>q^{2}+q$.

By Theorem 1, $f\left(q^{2}\right)<q^{2}+q+2$, and since $f\left(q^{2}\right)$ is an integer, $f\left(q^{2}\right)=$ $q^{2}+q+1$.

3. Ramsey graphs and block designs. This paper was motivated, to a great extent, by the work of Erdös, Rényi, and Sós [14], and especially by the following result now known as "the Friendship Theorem":

Proposition 1 (ERdös, RÉNYI, Sós). Let $G$ be a graph with $m$ vertices such that every pair of vertices is joined by exactly one path of length 2 in $G$. Then $m=2 k+1$ and we may number the vertices of $G$ as $v, a_{1}, \cdots, a_{k}, b_{1}, \cdots, b_{k}$ so that $v \sim a_{i}, v \sim b_{i}, a_{i} \sim b_{i}$ for $i=1,2, \cdots, k$ are all the adjacencies in $G$.

See [18],$[25]$ for elementary graph-theoretic proofs of this theorem.

If we define a " $\lambda$-friendship graph" $G$ to be a finite graph in which every pair of vertices is joined by exactly $\lambda$ paths of length 2 in $G$, then if $\lambda>1$, the graph $G$ must be regular of some valence $k$. This was proved by Erdös (see [1, p. 485]), and independently in a stronger form by Ryser [32, Theorem 2.1].

Ahrens and Szekeres [1] called such graphs having $v$ points, valence $k$, and $\lambda$ paths of length 2 joining any two points, $(v, k, \lambda)$-graphs. They generalized the Friendship Theorem by constructing nontrivial graphs with parameters $v=\lambda^{2}(\lambda+2)$, $k=\lambda(\lambda+1)$ for $\lambda$ any prime power. Rudvalis [31] has investigated $(v, k, \lambda)$ graphs, and has constructed several other nontrivial families of them.

Of course, for any $\lambda$ there is always the trivial $(\lambda+2, \lambda+1, \lambda)$-graph which is the complete graph on $\lambda+2$ points. Proposition 1 states that the only $(v, k, 1)$ graph is the trivial $(3,2,1)$-graph $K_{3}$. Ahrens and Szekeres remark that R. C. Bose has shown that for each $\lambda$ there are at most finitely many $(v, k, \lambda)$-graphs. Indeed, this follows easily from an eigenvalue argument similar to that used in the proof of our Lemma 4.

Let $g(\lambda, n)=R\left(K_{2, \lambda+1}, K_{1, n}\right)$. It is easy to modify the proof of Lemma 1 to show that $g(\lambda, n) \leqslant 1+n+1 / 2\left(\lambda+1+\sqrt{(\lambda-1)^{2}+4 \lambda n}\right.$, and that equality holds here if and only if there exists a $(v, k, \lambda)$-graph with $n=v-k$. In particular, $g(\lambda, v-k)=1+v$ whenever a $(v, k, \lambda)$-graph exists.

The construction of Lemma 6 can be generalized. Let $D=(V, B)$ be a 
$(v, k, \lambda)$-design such that $V=\left\{x_{1}, \cdots, x_{v}\right\}$ is a set of $v$ points and $B=\left\{B_{1}, \cdots, B_{v}\right\}$ is a set of $v$ blocks $B_{i}$ such that $B_{i} \subseteq V,\left|B_{i}\right|=k$, and $i \neq j$ implies $\left|B_{i} \cap B_{j}\right|=\lambda$ for all $i, j$. Suppose $D$ admits a "polarity" $\pi$, that is, a bijection $\pi: V \cup B \rightarrow$ $V \cup B$ such that $\pi(V)=B, \pi^{2}$ is the identity, and $x_{i} \in \pi\left(x_{j}\right)$ implies that $x_{j} \in$ $\pi\left(x_{i}\right)$ for all $i, j$. (See Rudvalis [31].) Then we may define a "polarity graph" $G(D, \pi)$ with vertex-set $V$ and $x_{i} \sim x_{j}$ if and only if $x_{i} \neq x_{j}$ and $x_{i} \in \pi\left(x_{j}\right)$.

We can characterize polarity graphs in terms of their local "common neighbor" structure, and we can show that if a $(v, k, \lambda)$-polarity graph exists, then $g(\lambda, v-k+1)$ is either $1+v$ or $2+v$. As a special case, if $B$ is a $(v, k, \lambda)$-difference set in an abelian group $(V,+)$, then letting $x \sim y$ if and only if $x \neq y$ and $x+y \in B$ defines a $(v, k, \lambda)$-polarity graph with vertex-set $V$.

Evidently there is an intimate connection between the Ramsey numbers $g(\lambda, n)$ and a large class of "classical" combinatorial designs. We outline this connection in our paper "Ramsey graphs and block designs" (to appear elsewhere), and we plan to study particular cases in sequels, the present paper being the first such sequel (corresponding to the $\left(q^{2}+q+1, q+1,1\right)$-designs arising from the finite Desarguesian projective planes).

\section{REFERENCES}

1. R. W. Ahrens and G. Szekeres, On a combinatorial generalization of 27 lines associated with a cubic surface, J. Austral. Math. Soc. 10 (1969), 485-492. MR 42 \#419.

2. J. A. Bondy and P. Erdös, Ramsey numbers for cycles in graphs, J. Combinatorial Theory Ser. (B) 14 (1973), 46-54. MR 47 \#6540.

3. W. G. Brown, On graphs that do not contain a Thomsen graph, Canad. Math. Bull. 9 (1966), 281-285. MR 34 \#81.

4. S. A. Burr and J. A. Roberts, On Ramsey numbers for stars, Utilitas Math. 4 (1973), 217-220.

5. G. Chartrand and S. Schuster, On the existence of specified cycles in complementary graphs, Bull. Amer. Math. Soc. 77 (1971), 995-998. MR 44 \#6527.

6. V. Chvátal, Hypergraphs and Ramseyian theorems, Proc. Amer. Math. Soc. 27 (1971), 434-440. MR $42 \# 5855$.

7. V. Chvátal and F. Harary, Generalized Ramsey theory for graphs, Bull. Amer. Math. Soc. 78 (1972), 423-426. MR $45 \# 110$.

8. - Generalized Ramsey theory for graphs. I. Diagonal numbers, Per. Math. Hungar. 3 (1973), 113-122.

9. - Generalized Ramsey theory for graphs. II. Small diagonal numbers, Proc. Amer. Math. Soc. 32 (1972), 389-394.

10. - Generalized Ramsey theory for graphs. III. Small off-diagonal numbers, Pacific J. Math. 41 (1972), 335-345. MR 47 \#3248.

11. E. J. Cockayne, An application of Ramsey's theorem, Canad. Math. Bull. 13 (1970), 145-146. MR 41 \#6721.

12. P. Erdös, Some remarks on the theory of graphs, Bull. Amer. Math. Soc. 53 (1947), 292-294. MR 8, 479.

13. P. Erdös and R. Rado, A partition calculus in set theory, Bull. Amer. Math. Soc. 62 (1956), 427-489. MR 18, 458.

14. P. Erdös, A. Rényi and V. T. Sós, On a problem of graph theory, Studia Sci. Math. Hungar. 1 (1966), 215-235. MR 36 \#6310. 
15. P. Erdös and G. Szekeres, A combinatorial problem in geometry, Compositio Math. 2 (1935), 463-470.

16. R. J. Faudree and R. H. Schelp, All Ramsey numbers for cycles in graphs, Discrete Math. 8 (1974), 313-329.

17. R. J. Faudree, S. L. Lawrence, T. D. Parsons and R. H. Schelp, Path-cycle Ramsey numbers, Discrete Math. 10 (1974), 269-277.

18. J. Galovich, S. Galovich and S. Schuster, An elementary proof of the Friendship Theorem (to appear).

19. L. Gerencsér and A. Gyárfás, On Ramsey-type problems, Ann. Univ. Sci. Budapest Eötövüs Sect. Math. 10 (1967), 167-170. MR 39 \#1351.

20. J. E. Graver and J. Yackel, Some graph theoretic results associated with Ramsey's Theorem, J. Combinatorial Theory 4 (1968), 125-175. MR 37 \#1278.

21. R. E. Greenwood and A. M. Gleason, Combinatorial relations and chromatic graphs, Canad. J. Math. 7 (1955), 1-7. MR 16, 733.

22. F. Harary, Graph theory, Addison-Wesley, Reading, Mass., 1969. MR 41 \#1566.

23. - Recent results on generalized Ramsey theory for graphs, Graph Theory and Applications, Springer-Verlag, Berlin, 1972, pp. 125-138.

24. J. G. Kalbfleisch, Upper bounds for some Ramsey numbers, J. Combinatorial Theory 2 (1967), 35-42. MR 35 \#2794.

25. J. Q. Longyear and T. D. Parsons, The friendship theorem, Nederl. Akad. Wetensch. Proc. Ser. A 75 = Indag. Math. 34 (1972), 257-262. MR 46 \#5169.

26. T. D. Parsons, The Ramsey numbers $r\left(P_{m}, K_{n}\right)$, Discrete Math. $6(1973), 159-162$. MR 48 \#150.

27. - Path-star Ramsey numbers, J. Combinatorial Theory Ser. B 17 (1974), $51-58$.

28. F. P. Ramsey, On a problem of formal logic, Proc. London Math. Soc. 30 (1930), 264-286.

29. V. Rosta, On a Ramsey type problem of J. A. Bondy and P. Erdös. I, II, J. Combinatorial Theory Ser. B 25 (1973), 94-104, 105-120.

30. B. L. Rothschild, A generalization of Ramsey's theorem, Proc. Sympos. Pure Math., vol. 19, Amer. Math. Soc., Providence, R. I., 1971, pp. 205-213.

31. A. Rudvalis, $(v, k, \lambda)$-graphs and polarities of $(v, k, \lambda)$-designs, Math. Z. 120 (1971), 224-230. MR 45 \#3220.

32. H. J. Ryser, $A$ generalization of the matrix equation $A^{2}=J$, Linear Algebra and Appl. 3 (1970), 451-460. MR 42 \#569.

33. S. Schuster, Progress on the problem of eccentric hosts, Graph Theory and Applications, Springer-Verlag, Berlin, 1972, pp. 283-290.

34. H. L. Skala, A variation of the Friendship Theorem, SIAM J. Appl. Math. 23 (1972), 214-220.

35. K. Walker, Dichromatic graphs and Ramsey numbers, J. Combinatorial Theory 5 (1968), 238-243. MR 38 \#79.

36. J. E. Williamson, A Ramsey-type problem for paths in digraphs, Math. Ann. 203 (1973), 117-118.

DEPARTMENT OF MATHEMATICS, PENNSYLVANIA STATE UNIVERSITY, UNIVERSITY PARK, PENNSYLVANIA 16802 